\title{
Forecast of Power System Load in Short Term
}

\author{
Hengshu Ye
}

School of Environmental Science and Technology, North China Electric Power University, Baoding 071000, China.

\section{4@qq.com}

Keywords: Sustainable Curve of Electric Power Charge, ARIMA, Regression Analysis

\begin{abstract}
In this paper, according to large quantity of historical statistics, we have established a model which could successfully forecast the power charge in two regions. Because of the different efforts between weekdays, weekends and holidays, we made a piecewise function ${ }^{[1]}$ to decrease the error. The method of 2 times curve fitting was used to analyze the electric power charge of minimum and average per day by Matlab. Then an ARIMA (Auto Regressive Integrated Moving Average Model) connected with statistics between 2009 to 2014 was set up and verified available residual analysis. We also take climate factors into consideration. Being supported by huge data the model can predict variation tendency of electric power charge efficiently.
\end{abstract}

\section{Introduction}

The short-term load forecasting is the foundation of electric power system's function and analysis. Improving accuracy of it is an important point to optimize the scientific nature of the strategic decision. In modern power system, the electrical appliances are various, and so many factors can exert an effect on the load. Now we have gathered around numerous information from 1st January, 2009 to 10th January, 2015 in two regions.

\section{The Data Processing}

We used Excel to calculate two regions' charge of maximum, minimum and average each day, and draw curve graphs just as follows:[x-axis is time(day), $y$-axis is load(MW)]

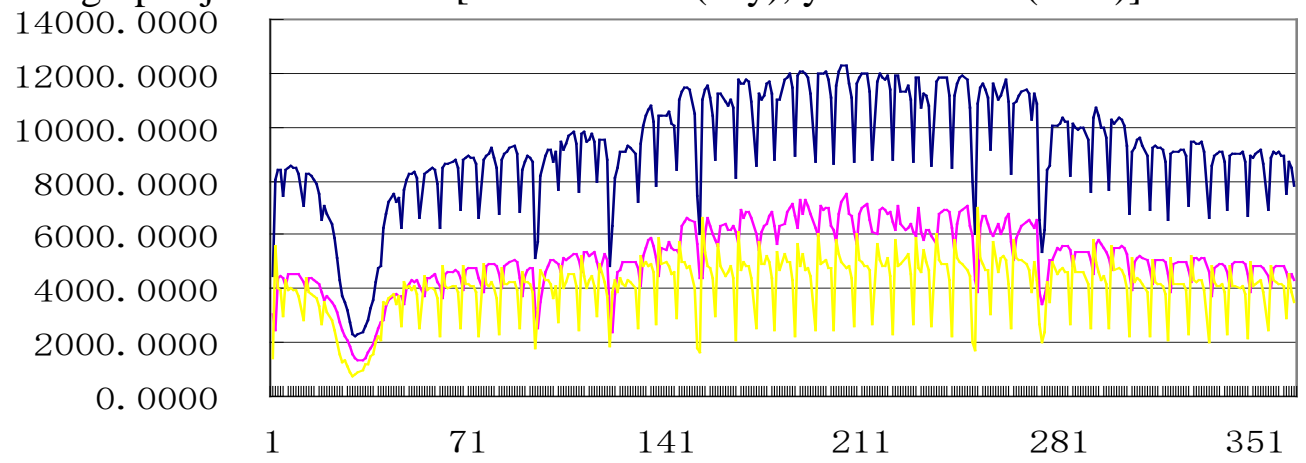

Figure 1 Time-Load relations between two regions

From the graph above, we can reach a conclusion that summer is the fastigium during the whole year.

\section{The modeling Process}

\subsection{Data pretreatment}

As for these data, we set up ARIMA(n, m) to analyze:

$$
x_{t}=\sum_{i=1}^{n} \varphi_{i} x_{t-i}+\alpha_{t}-\sum_{j=1}^{m} \theta_{j} \alpha_{t-j}
$$

$\varphi_{i}(i=1,2, \cdots, n)$ :Auto Regressive parameters; 
$\theta_{j}(j=1,2, \cdots, m)$ :Moving average parameters;

$\left\{\alpha_{t}\right\}$ :Zero-mean white noise source, the variance is $\sigma_{a}^{2}$;

Particularly, when m=0, ARIMA(n,m) is equal to Auto Regressive model AR(n).

$$
x_{t}=\sum_{i=1}^{n} \varphi_{i} x_{t-i}+\alpha_{t}
$$

Standardized:

$$
x_{t}=\frac{x_{t}^{(0)}-\mu_{x}}{\sigma_{x}^{2}}
$$

Particularly,

$$
\begin{aligned}
& \mu_{x}=\frac{1}{N} \sum_{t=1}^{N} x_{t}^{(0)} \\
& \sigma_{x}^{2}=\frac{1}{N-1} \sum_{t=1}^{N}\left(x_{t}^{(0)}-\mu_{x}\right)^{2}
\end{aligned}
$$

Then we get the prediction formula:

$$
x_{t}=\varphi_{1} x_{t-1}+\varphi_{2} x_{t-2}+\varphi_{3} x_{t-3}+\varphi_{4} x_{t-4}+\varphi_{5} x_{t-5}+\alpha_{t}-\theta_{1} \alpha_{t-1}-\theta_{2} \alpha_{t-2}-\theta_{3} \alpha_{t-3}-\theta_{4} \alpha_{t-4}
$$

According to the difference formula $\omega_{t}=\nabla^{d} Z_{t}$, we converse non-linear stochastic variation to zero-mean stationary random sequence[2] through stationary treatment. The auto correlation function attenuats fast after the difference. We used ' $d$ ' as the difference order, and in this model, $d$ is equal to 2 .

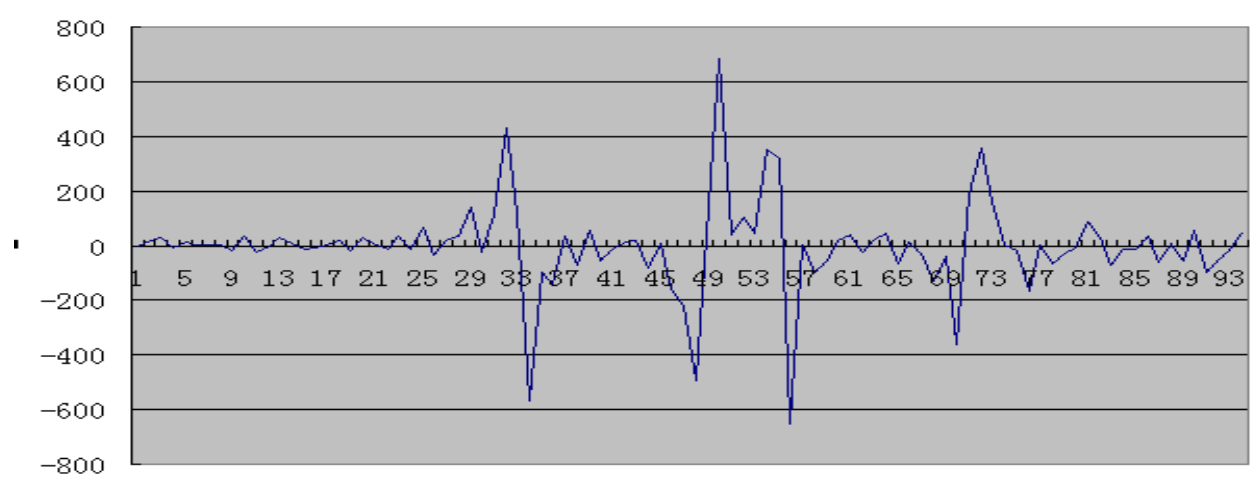

Figure 2 Curves after two differences

\subsection{AR Model order}

We decided to fit MA(1), MA(2), namely, ARIMA(2, 1), ARIMA(2, 2) into curves:

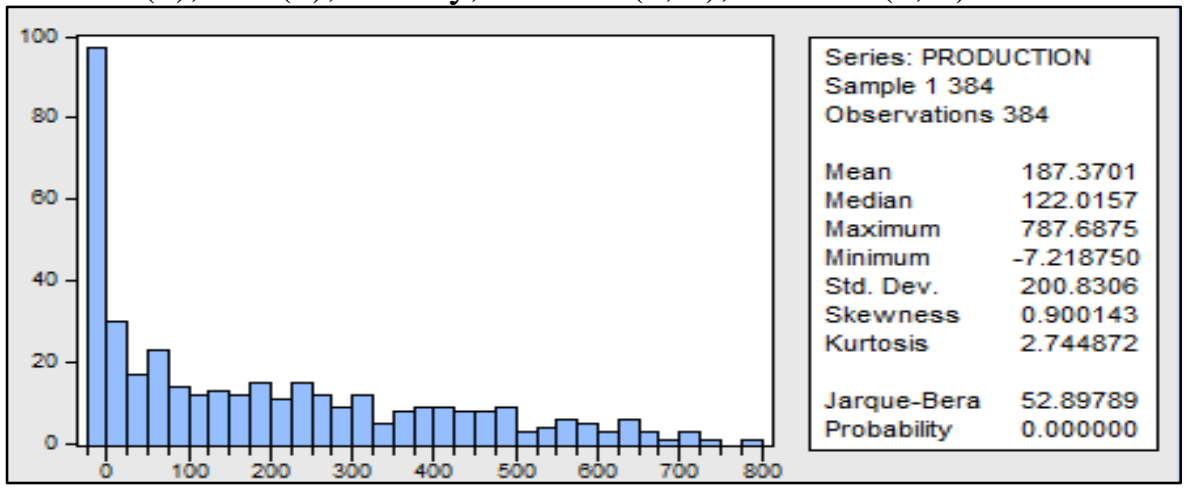

Figure 3

This is the sequence of zero-mean white noise station. 


\subsection{Model Parameter Estimation}

Based on the AR model order and relations above, we choose the best model to estimate the parameters by SPSS ${ }^{[3]}$ :

\begin{tabular}{|crrrr|}
\hline MA Backcast: 3 5 & & & & \\
\hline \hline Variable & Coefficient & Std. Error & t-Statistic & Prob. \\
\hline \hline AR(1) & -0.524887 & 0.133066 & -3.944552 & 0.0001 \\
AR(2) & 0.989831 & 0.164477 & 6.018068 & 0.0000 \\
AR(3) & 0.421559 & 0.121821 & 3.460479 & 0.0006 \\
AR(4) & -0.147125 & 0.152302 & -0.966006 & 0.3347 \\
AR(5) & 0.174372 & 0.072386 & 2.408909 & 0.0165 \\
MA(1) & 1.129172 & 0.130734 & 8.637152 & 0.0000 \\
MA(2) & -0.197931 & 0.219944 & -0.899919 & 0.3687 \\
MA(3) & -0.466474 & 0.130499 & -3.574528 & 0.0004 \\
\hline \hline
\end{tabular}

Figure 4

Finally, we get the ARIMA model:

$$
x_{t}=-0.52487 x_{t-1}+0.989831 x_{t-2}+\alpha_{t}-1.129172 \alpha_{t-1}+0.197931 \alpha_{t-2}
$$

\subsection{Modeling Checking}

\begin{tabular}{|c|c|c|c|c|c|c|c|c|}
\hline Autocor & relation & Partial Co & orrelation & & $A C$ & PAC & Q-Stat & Prob \\
\hline 1 & 1 & 1 & 1 & 1 & -0.007 & -0.007 & 0.0163 & \\
\hline 1 & 1 & & 1 & 2 & 0.015 & 0.015 & 0.0998 & \\
\hline 1 & 1 & 1 & 1 & 3 & -0.022 & -0.022 & 0.2916 & \\
\hline 1 & 1 & 11 & 1 & 4 & -0.010 & -0.011 & 0.3333 & \\
\hline 1 & ין & 1 & ין & 5 & 0.074 & 0.075 & 2.4474 & \\
\hline 1 & 1 & $1)$ & 1 & 6 & 0.019 & 0.020 & 2.5849 & \\
\hline 1 & 1 & 14 & 1 & 7 & -0.055 & -0.058 & 3.7536 & \\
\hline 1 & 1 & 11 & 1 & 8 & -0.014 & -0.012 & 3.8325 & \\
\hline II & 1 & 11 & 1 & 9 & -0.029 & -0.025 & 4.1532 & 0.042 \\
\hline 1 & 1 & II & 1 & 10 & -0.007 & -0.015 & 4.1723 & 0.124 \\
\hline 1 & ון & & וק & 11 & 0.029 & 0.026 & 4.5102 & 0.211 \\
\hline IL & 1 & II & 1 & 12 & -0.045 & -0.038 & 5.3159 & 0.256 \\
\hline IL & 1 & II) & 1 & 13 & -0.026 & -0.025 & 5.5893 & 0.348 \\
\hline 1 & ין & 1 & ין & 14 & 0.051 & 0.055 & 6.6001 & 0.359 \\
\hline 1 & 1 & II) & 1 & 15 & -0.035 & -0.034 & 7.0800 & 0.421 \\
\hline 15 & 1 & 回 & 1 & 16 & -0.054 & -0.067 & 8.2564 & 0.409 \\
\hline 1 & 1 & $1)$ & 1 & 17 & 0.005 & 0.011 & 8.2649 & 0.508 \\
\hline I & 1 & 10 & ין & 18 & 0.020 & 0.030 & 8.4202 & 0.588 \\
\hline 1 & ין & 17 & ין & 19 & 0.060 & 0.045 & 9.8446 & 0.544 \\
\hline
\end{tabular}

Figure 5

\section{Summary}

This model could forecast the electric power charge each day accurately. It is near the practical data with the support of historical statistics. However, we ignore the climate factors and made a hypothesis that the information are all correct and reliable. These are limitations of the model.

\section{References}

[1] Nihuan Liao.Overview on the Short Term Forecast in Electric Power System.The Protection and Control of Power System.2011.1(1):2-6. 
[2] Chongqing Kang, Niande Xiang, Qing Xia.The Load Derivation in Short Term Forecasting Using Weather Factor[J].Journal of Electric Power System and Automation System. 2006,10(5):70-82.

[3] Chongqing Kang, Anshi Zhou. Practice of Load Derivation for Super-short Load Forecast in Power System[J].The Grid Technology,2006,4(7):6-10. 